\title{
Correction: Regev, Y., et al. Molecular Identification and Characterization of Vibrio Species and Mycobacterium Species in Wild and Cultured Marine Fish from the Eastern Mediterranean Sea. Microorganisms 2020, 8, 863
}

\author{
Yael Regev ${ }^{1,2}$, Nadav Davidovich ${ }^{3}{ }^{(0)}$, Ran Berzak ${ }^{1,2}$, Stanley C. K. Lau ${ }^{4}$, Aviad P. Scheinin ${ }^{1,2}$, \\ Dan Tchernov ${ }^{1,2}$ and Danny Morick ${ }^{1,2, *}$ \\ 1 Department of Marine Biology, Leon H. Charney School of Marine Sciences, University of Haifa, \\ Haifa 3498838, Israel; yaelchac@gmail.com (Y.R.); ranberzak@gmail.com (R.B.); \\ shani.aviad@gmail.com (A.P.S.); dtchernov@univ.haifa.ac.il (D.T.) \\ 2 Morris Kahn Marine Research Station, University of Haifa, Haifa 3498838, Israel \\ 3 Israeli Veterinary Services, Bet Dagan 5025001, Israel; Nadavd@moag.gov.il \\ 4 Department of Ocean Science, The Hong Kong University of Science and Technology, Clear Water Bay, \\ Kowloon, Hong Kong; scklau@ust.hk \\ * Correspondence: dmorick@univ.haifa.ac.il
}

\section{Change in Affiliations}

We would like to change the authors' affiliation of paper [1] from:

Yael Regev ${ }^{1,2}{ }^{2}$ Nadav Davidovich ${ }^{3}$, Ran Berzak ${ }^{1,2}$, Stanley C. K. Lau ${ }^{4}$, Aviad P. Scheinin ${ }^{1,2}$, Dan Tchernov ${ }^{1,2}$ and Danny Morick ${ }^{1,2, *}$

1 Department of Marine Biology, Leon H. Charney School of Marine Sciences, University of Haifa, Haifa 3498838, Israel; yaelchac@gmail.com (Y.R.); ranberzak@gmail.com (R.B.); shani.aviad@gmail.com (A.P.S.); dtchernov@univ.haifa.ac.il (D.T.)

2 Morris Kahn Marine Research Station, University of Haifa, Haifa 3498838, Israel

$3 \quad$ Israeli Veterinary Services, Bet Dagan 5025001, Israel; Nadavd@moag.gov.il

4 Department of Ocean Science, The Hong Kong University of Science and Technology, Clear Water Bay, Kowloon, Hong Kong; scklau@ust.hk

* Correspondence: dmorick@univ.haifa.ac.il

to the correct version, as follows:

Yael Regev ${ }^{1,2}$, Nadav Davidovich ${ }^{3}$, Ran Berzak ${ }^{1,2}$, Stanley C. K. Lau ${ }^{4,5}$, Aviad P. Scheinin ${ }^{1,2}$, Dan Tchernov ${ }^{1,2,5}$ and Danny Morick $1,2,5, *$

1 Department of Marine Biology, Leon H. Charney School of Marine Sciences, University of Haifa, Haifa 3498838, Israel; yaelchac@gmail.com (Y.R.); ranberzak@gmail.com (R.B.); shani.aviad@gmail.com (A.P.S.); dtchernov@univ.haifa.ac.il (D.T.)

2 Morris Kahn Marine Research Station, University of Haifa, Haifa 3498838, Israel

3 Israeli Veterinary Services, Bet Dagan 5025001, Israel; Nadavd@moag.gov.il

4 Department of Ocean Science, The Hong Kong University of Science and Technology, Clear Water Bay, Kowloon, Hong Kong; scklau@ust.hk 
5 Hong Kong Branch of Southern Marine Science and Engineering Guangdong Laboratory (Guangzhou), Hong Kong, China

* Correspondence: dmorick@univ.haifa.ac.il

We apologize for any inconvenience caused to the readers.

Conflicts of Interest: The authors declare no conflict of interest.

\section{Reference}

1. Regev, Y.; Davidovich, N.; Berzak, R.; Lau, S.C.K.; Scheinin, A.P.; Tchernov, D.; Morick, D. Molecular Identification and Characterization of Vibrio Species and Mycobacterium Species in Wild and Cultured Marine Fish from the Eastern Mediterranean Sea. Microorganisms 2020, 8, 863. [CrossRef] 